\title{
Análisis intercultural del trastorno por uso de alcohol: criterios diagnósticos en universitarios
}

Agosto 2020, Vol. 12, $\mathrm{N}^{\circ} 2,1-10$

revistas.unc.edu.ar/inde x.php/racc

Conde, Karina*,a; Gimenez, Paula Victoriaa; Díaz-Castela, María del Marº Ortega-Martinez, Ana

Cremonte, Mariana ${ }^{a}$

\section{Artículo Original}

\section{Resumen}

El objetivo de este estudio fue examinar las manifestaciones clínicas del trastorno por uso de alcohol (TUA) en universitarios de países con distintas prácticas de consumo, y estimar el potencial de una medida de consumo de alcohol como marcador de TUA. Participaron estudiantes de universidades en Argentina $(N=2157)$, España $(N=320)$ y Brasil $(N=149)$. Se evaluaron criterios diagnósticos de TUA, puntos de corte compatibles con el DSM 5, y una medida de consumo excesivo episódico de alcohol. Se realizaron análisis descriptivos y de clases latentes. Generalmente el TUA grave se caracterizó por endorsar todos los criterios; el moderado-leve por tolerancia, pérdida de control, problemas interpersonales y negligencia de obligaciones. En los países con un patrón húmedo de consumo (Argentina y España) los criterios uso en situaciones peligrosas y consumo a pesar de problemas mentales/físicos se manifestaron en categorías distintas. La medida de consumo presentó potencial como marcador de TUA
Palabras clave:

consumo de alcohol, DSM 5, criterios de trastorno por uso de alcohol, análisis intercultural, jóvenes.
Abstract

Cross-Cultural Analysis of Diagnostic Criteria for Alcohol Use Disorder in Young University Students. This study aimed to examine the clinical manifestations of Alcohol Use Disorder (AUD) in university students from countries with different drinking practices, and the potential of a drinking measure as an AUD marker. University students from Argentina $(N=2157)$, Spain $(N=320)$, and Brazil $(N=149)$ participated in the study. The TUA criteria and the cut off-points were compatible with the DSM 5, and a measure of binge drinking was evaluated. Descriptive and latent class analyses were performed. In general, the severe AUD is characterized by the endorsement of all criteria; the moderate-mild, by tolerance, loss of control (quit/cut), interpersonal problems, and role impairment. In countries with a wet drinking pattern (Argentina and Spain), the criteria of hazardous use and psychological or physical problems due to use were characteristic of different severity levels. The binge drinking measure showed potential as a marker of TUA.

Keywords:

alcohol consumption, DSM 5, alcohol use disorder criteria, cross-cultural analysis, youth.

Recibido el 16 de septiembre de 2019; Aceptado el 19 de febrero de 2020

Editaron este artículo: Silvana Montes, Paula Abate, Gabriela Raynaudo y Yanina Michelini

\section{Introducción}

El ingreso a la universidad se traduce en cambios en la vida de los jóvenes; uno de ellos es el modo en que consumen alcohol. Los universitarios presentan tasas de consumo más altas que la población general (Slutske, 2005), e incluso el ingreso a la universidad implica un aumento en el consumo (Caldeira et al., 2009). Además, el uso de alcohol en la juventud produce efectos que se extienden hasta la adultez, y predispone al desarrollo de un trastorno por uso de alcohol (TUA) (Zeigler et al., 2005).

El manual diagnóstico y estadístico de los

\footnotetext{
a Universidad Nacional de Mar del Plata, Instituto de Psicología Básica, Aplicada y Tecnología (IPSIBAT), Consejo de Investigaciones Científicas y Técnicas, Argentina.

b Universidad de Jaén, Departamento de Psicología, Jaén, España.

"Enviar correspondencia a: Conde, K. E-mail: kconde@mdp.edu.ar
}

Citar este artículo como: Conde, K., Gimenez, P. V., Díaz-Castela, M del M., Ortega-Martinez, A., \& Cremonte, Mariana. (2020). Análisis intercultural del trastorno por uso del alcohol: criterios diagnósticos en universitarios. Revista Argentina de Ciencias del Comportamiento, 12(2), 1-10 
trastornos mentales (DSM), en su versión anterior (DSM-IV), distinguía dos tipos de TUAs: el abuso y la dependencia (American Psychiatric Association, 2000). Sin embargo, debido a que algunas investigaciones demostraron que este trastorno presentaría una única dimensión (Bond et al., 2012), en la quinta edición del DSM se estableció que la suma de criterios conformaba un único TUA con tres niveles de severidad (i.e. leve, moderado o grave) según la cantidad de criterios diagnósticos cumplidos, sin importar cuáles fueran (American Psychiatric Association, 2013).

Uno de los puntos controversiales referidos a este modelo reside en que los criterios diagnósticos sólo consideran los problemas y comportamientos asociados al consumo, pero no tienen en cuenta patrones de ingesta de alcohol. Algunos han dudado de la fiabilidad y validez de un criterio de diagnóstico que tenga en cuenta el nivel de consumo de alcohol (Dawson, 2000) y otros han dado cuenta de su importancia diagnóstica, ya que informaría sobre la etapa del trastorno (Li, Hewitt, \& Grant, 2007) y permitirían un mejor ajuste del modelo de una única dimensión (Borges et al., 2010). Una medida de consumo de alcohol que se ha propuesto como posible indicador es el consumo excesivo episódico, considerado como la ingesta de aproximadamente cinco o más consumiciones en una única ocasión (Saha, Stinson, \& Grant, 2007). Este patrón es característico de los estudiantes universitarios a nivel mundial (Karam, Kypri, \& Salamoun, 2007).

Otra controversia corresponde a la detección de personas con sólo un problema por el consumo de alcohol, antes conocidos como huérfanos diagnósticos. Esta es una categoría que engloba a aquellas personas que no cumplen la cantidad mínima de criterios (dos) para considerar un TUA, pero que presentan problemas asociados al consumo (Gilbert \& Marzell, 2018). En los jóvenes, la prevalencia de personas con problemas por uso de alcohol y sin diagnóstico es alta (Hagman, 2018) y muchos de ellos progresarían hacia la dependencia en la adultez (Grabitz et al., 2012).

Por lo expuesto, es necesario desarrollar procedimientos eficaces de detección precoz del TUA en los jóvenes y que permitan además identificar el grado de severidad. Si bien se realizaron estudios recientes que indicarían que algunos criterios diagnósticos se asocian a niveles de mayor severidad (Swift et al., 2016), no se hallaron antecedentes en habla hispana y en población joven que estimen la relación entre la refrendación de cada criterio y el nivel de severidad de TUA (leve, moderado y grave) o de haber padecido únicamente un problema por uso de alcohol.

Por ello, el objetivo de esta investigación será examinar las manifestaciones clínicas del TUA en tres grupos de estudiantes universitarios pertenecientes a países con distintas prácticas de consumo de alcohol: Argentina, España y Brasil. Para ello se determinará la probabilidad de que cada criterio diagnostico sea refrendado por quienes pertenecen a cada una de las clases conformadas según los niveles de diagnóstico del TUA. Además, se estimará el potencial de una medida de consumo excesivo episódico para la detección de la severidad del TUA. El modelo será testeado en jóvenes estudiantes universitarios de tres países: Argentina, España y Brasil, cuyo consumo de alcohol presenta particularidades y desafíos a la salud pública (Hingson, Zha, \& Weitzman, 2009). Si bien estos países comparten ciertas características, difieren en su idioma, su nivel de ingresos, y en sus patrones de consumo culturales (Savic, Room, Mugavin, Pennay, \& Livingston, 2016), lo que resulta de especial interés, ya que en el análisis no sólo se podrán tener en cuenta los patrones individuales de consumo excesivo episódico, sino también observar cómo se dan las manifestaciones según el contexto.

\section{Método}

\section{Participantes}

Este estudio cuenta con datos de estudiantes universitarios de los primeros tres años de universidades en Argentina, España y Brasil. La muestra total estuvo constituida por un total de 2626 estudiantes universitarios de primer a tercer año. De estos, 2157 eran estudiantes de las diferentes titulaciones de una universidad pública del Mar del Plata (Argentina); 320 eran estudiantes de la titulación de Psicología en una universidad pública de Jaén (España) y 149 fueron estudiantes de diferentes universidades públicas de Brasil. En el caso de Argentina y España se utilizó un muestreo probabilístico. En Argentina se realizó un muestreo aleatorio por conglomerados, seleccionando aleatoriamente cursos de todas las carreras e invitando a participar a todos los alumnos de los cursos 
Conde, K. et al. / RACC, 2020, Vol. 12, №2, 1-10

seleccionados. En España se entrevistó a todos conveniencia. Los datos fueron obtenidos entre los estudiantes de los cursos de Psicología, los años 2008 y 2014.

mientras que en Brasil la muestra se obtuvo por

Tabla 1.

Características de las muestras, estudiantes universitarios de Argentina, España y Brasil

\begin{tabular}{|c|c|c|c|c|c|c|c|c|c|}
\hline \multirow[b]{2}{*}{ Indicadores } & \multicolumn{3}{|c|}{ Argentina $(N=2157)$} & \multicolumn{3}{|c|}{ España $(N=320)$} & \multicolumn{3}{|c|}{ Brasil $(N=149)$} \\
\hline & $\%$ & $M(D S)$ & IC 95\% & $\%$ & $M(D S)$ & IC 95\% & $\%$ & $M(D S)$ & IC $95 \%$ \\
\hline \multicolumn{10}{|l|}{ Sexo } \\
\hline Femenino & 63 & & $61-65$ & 83 & & $79-88$ & 76 & & $68-83$ \\
\hline Masculino & 37 & & $35-39$ & 17 & & $12-21$ & 24 & & $17-31$ \\
\hline Edad & & $\begin{array}{l}22.05 \\
(6.05)\end{array}$ & $\begin{array}{l}21.79- \\
22.32\end{array}$ & & $\begin{array}{l}20.25 \\
(4.14)\end{array}$ & $\begin{array}{l}19.86- \\
20.69\end{array}$ & & $\begin{array}{l}23.32 \\
(5.63)\end{array}$ & $\begin{array}{l}22.46- \\
24.20\end{array}$ \\
\hline \multicolumn{10}{|l|}{ Consumo de alcohol } \\
\hline Prevalencia de vida & 94 & & $93-95$ & 97 & & $95-99$ & 87 & & $81-91$ \\
\hline Último año & 90 & & $89-91$ & 96 & & $93-98$ & 77 & & $70-83$ \\
\hline CEEA & 43 & & $40-45$ & 54 & & $48-59$ & 30 & & $23-38$ \\
\hline \multicolumn{10}{|l|}{ Criterios diagnósticos } \\
\hline Síntomas de abstinencia & 6 & & $5-7$ & 6 & & $3-9$ & 7 & & $3-12$ \\
\hline Apetencia de la sustancia & 3 & & $2-4$ & 2 & & $1-3$ & 9 & & $5-14$ \\
\hline Tolerancia & 12 & & $10-13$ & 17 & & $13-21$ & 11 & & $5-15$ \\
\hline Descuido de actividades & 1 & & $1-2$ & 1 & & $0-2$ & 6 & & $3-11$ \\
\hline Tiempo empleado & 7 & & $6-8$ & 12 & & $9-16$ & 6 & & $3-11$ \\
\hline Mayor consumo & 3 & & $2-4$ & 3 & & $1-5$ & 6 & & $3-10$ \\
\hline Pérdida de control & 16 & & $15-18$ & 20 & & $16-24$ & 45 & & $38-52$ \\
\hline $\begin{array}{l}\text { Consumo a pesar de } \\
\text { problemas mentales/físicos }\end{array}$ & 11 & & $9-12$ & 13 & & $10-17$ & 18 & & $12-24$ \\
\hline $\begin{array}{l}\text { Uso en situaciones } \\
\text { físicamente peligrosas }\end{array}$ & 8 & & $7-9$ & 20 & & $16-24$ & 5 & & $2-9$ \\
\hline Problemas interpersonales & 10 & & $9-12$ & 22 & & $18-27$ & 7 & & $3-11$ \\
\hline $\begin{array}{l}\text { Negligencia de } \\
\text { obligaciones prioritarias } \\
\text { Trastorno por uso de alcohol }\end{array}$ & 24 & & $22-26$ & 22 & & $18-27$ & 11 & & $6-16$ \\
\hline Negativo & 56 & & $54-58$ & 44 & & $39-50$ & 44 & & $37-52$ \\
\hline Un problema & 19 & & $17-20$ & 17 & & $13-21$ & 26 & & $19-33$ \\
\hline Leve & 17 & & $16-19$ & 25 & & $21-30$ & 18 & & $12-25$ \\
\hline Moderado & 6 & & $5-7$ & 12 & & $8-15$ & 7 & & $3-12$ \\
\hline Grave & 2 & & $2-3$ & 2 & & $1-3$ & 4 & & $1-7$ \\
\hline
\end{tabular}

Nota. IC = Intervalo de Confianza; CEEA = consumo excesivo episódico de alcohol

En todos los casos se utilizó un cuestionario auto-administrado estructurado con respuestas anónimas, ya utilizado en otras investigaciones. No hubo vínculo entre los encargados de la recolección de datos y los participantes; de esta forma, se intentaron minimizar los sesgos de clasificación.

\section{Diseño y Procedimiento}


Conde, K. et al. / RACC, 2020, Vol. 12, №2, 1-10

Instrumentos.

Los criterios para las medidas utilizadas en este estudio no experimental correlacional fueron:

Criterios de diagnóstico y trastorno por uso de alcohol. Se clasificaron de acuerdo con el DSM 5, utilizando la Sección de Alcohol de la Entrevista de Diagnóstico Internacional Compuesta (CIDI), que ha demostrado un buen funcionamiento psicométrico en distintos contextos, incluidos los abordados en este estudio (Kessler \& Üstün, 2004). En cuanto a los criterios de diagnóstico, se confeccionaron variables dicotómicas (sí/no) con cada uno de los 11 criterios que componen el TUA: síntomas de abstinencia, apetencia de la sustancia (craving), tolerancia, descuido de actividades importantes, tiempo empleado con consumir/adquirir la sustancia, consumo de mayores cantidades o por más tiempo del pretendido, pérdida de control en el consumo (no ser capaz de reducir o parar), consumo de alcohol a pesar de notar problemas mentales/físicos, uso en situaciones físicamente peligrosas, problemas interpersonales debidos al uso, y haber consumido o padecer las consecuencias del consumo durante la realización de obligaciones prioritarias. Los participantes fueron clasificados, en función de la cantidad de criterios de diagnóstico cumplidos en los últimos 12 meses, en las siguientes categorías diagnósticas: negativo (ningún criterio), un problema (1), TUA leve (2-3), moderado (4-5) y grave (6-11). Estos umbrales y criterios coinciden con los propuestos para la última versión del DSM 5 (American Psychiatric Association, 2013).

Consumo excesivo episódico de alcohol (CEEA). Fue definido como el consumo de cinco o más unidades estándar (11 gr aproximados de alcohol puro) de bebidas alcohólicas en una ocasión, con una frecuencia de, al menos, una vez al mes durante el último año (variable dicotómica sí/no).

\section{Procedimiento.}

El cuestionario fue administrado en las aulas de las asignaturas y en el horario de clase, con excepción de la muestra brasileña, en la que se administró mediante la plataforma SurveyMonkey. Cabe aclarar que estudios previos en poblaciones similares han evidenciado la precisión y comparabilidad de los datos obtenidos de estas formas (Conde \& Cremonte, 2015). La administración del cuestionario tuvo una duración aproximada de 20 minutos. La participación fue voluntaria (los estudiantes podían entregar el cuestionario en blanco o retirarse sin recibirlo) anónima, con consentimiento informado y sin compensación. Estuvieron disponibles investigadores entrenados para responder eventuales dudas durante la administración, y se dejó a disposición el contacto de los responsables del estudio. Se contó con avales del Comité de Ética del Instituto Nacional de Epidemiología y del Comité de Ética de la Universidad de Jaén.

\section{Análisis de datos}

Con el fin de estimar la probabilidad de quienes presentaban cada criterio, categoría de diagnóstico, y/o consumo excesivo de pertenecer a cada clase, se realizaron análisis de clases latentes (ACL). Este tipo de análisis permite una clasificación en base a la persona y según criterios probabilísticos y se ha empleado para explorar la relación entre patrones de consumo y TUA (Jackson et al., 2014). Se incluyeron las cinco categorías diagnósticas (negativo, un problema, leve, moderado y grave), cada uno de los 11 criterios (sí/no) y la presencia/ausencia de CEEA. Para determinar el número de clases a retener en cada muestra se utilizaron las medidas de parsimonia Akaike Information Criterion (AIC), el Bayesian Information Criterion ajustado al tamaño de la muestra $(\mathrm{aBIC})$, y el índice de entropía calculado en base a Zhang, Abarda, Contractor, Wang y Dayton (2018), prefiriendo aquel modelo que minimizara los valores del aBIC y AIC y maximizara el de entropía, en adición a la interpretabilidad. Para evitar el problema de los máximos locales, cada modelo fue reproducido 50 veces hasta encontrar el máximo global. Se ajustaron modelos de 2 a 5 clases con el paquete poLCA (Linzer \& Lewis, 2011) del software R 3.5.3. para Windows. Los datos perdidos (no mayores al $16 \%$ en las variables) tuvieron un procedimiento de imputación múltiple con el paquete MICE (Buuren \& Groothuis-Oudshoorn, 2011) del mismo software. Las proporciones de casos positivos en las variables no se vieron modificadas a pesar de ello.

\section{Resultados}

\section{Definición de Clases según Criterios de} Diagnóstico, Niveles de Trastorno y CEEA

En todos los contextos, el modelo con mejor ajuste fue el de cinco clases según los criterios AIC y aBIC (Tabla 2). Por otro lado, 
Conde, K. et al. / RACC, 2020, Vol. 12, №2, 1-10

independientemente del número de clases, el valor de la entropía fue superior a .90 (no se muestra en tablas).

Tabla 2.

Comparación del ajuste de los modelos del análisis de clases latentes para los criterios de diagnóstico, niveles de trastorno y consumo excesivo episódico en estudiantes universitarios de Argentina $(N=$ 2157), España $(N=320)$ y Brasil $(N=149)$

\begin{tabular}{|c|c|c|c|c|c|c|c|c|c|c|c|c|}
\hline \multirow[b]{2}{*}{ Modelo } & \multicolumn{4}{|c|}{ Argentina } & \multicolumn{4}{|c|}{ España } & \multicolumn{4}{|c|}{ Brasil } \\
\hline & $\begin{array}{c}\text { Log- } \\
\text { likelihood }\end{array}$ & Par. & AIC & $\mathrm{aBIC}$ & $\begin{array}{c}\text { Log- } \\
\text { likelihood }\end{array}$ & Par. & AIC & $\mathrm{aBIC}$ & $\begin{array}{c}\text { Log- } \\
\text { likelihood }\end{array}$ & Par. & $\mathrm{AIC}$ & $\mathrm{aBIC}$ \\
\hline $\begin{array}{c}2 \\
\text { clases }\end{array}$ & -8610.66 & 33 & 17287.31 & 17369.79 & -1519.26 & 33 & 3.104 .523 & 3124.21 & -647.94 & 33 & 1.361 .886 & 1356.58 \\
\hline $\begin{array}{c}3 \\
\text { clases }\end{array}$ & -7984.25 & 50 & 16068.51 & 16193.48 & -1386.90 & 50 & 2873.80 & 2903.63 & -574.02 & 50 & 1248.03 & 1239.99 \\
\hline $\begin{array}{c}4 \\
\text { clases }\end{array}$ & -7740.32 & 67 & 15614.65 & 15782.11 & -1323.72 & 67 & 2781.45 & 2821.41 & -544.81 & 67 & 1223.62 & 1212.85 \\
\hline $\begin{array}{c}5 \\
\text { clases }\end{array}$ & -7556.1 & 84 & 15280.2 & 15490.14 & -1292.38 & 84 & 2752.76 & 2802.87 & -519.61 & 84 & 1207.23 & 1193.72 \\
\hline
\end{tabular}

Las proporciones estimadas coincidieron en todos los casos con la membresía predicha por probabilidad posterior. En Argentina fueron: Clase $1=56 \%$, Clase $2=19 \%$, Clase $3=17 \%$, Clase 4 $=6 \%$, Clase $5=2 \%$. Para los estudiantes de España fueron: Clase $1=44 \%$, Clase $2=17 \%$, Clase $3=11 \%$, Clase $4=17 \%$, Clase $5=11 \%$. Finalmente, en Brasil se distribuyeron de la siguiente forma: Clase $1=44 \%$, Clase $2=26 \%$, Clase $3=15 \%$, Clase $4=10 \%$, Clase $5=04 \%$. Para todos los casos, la Clase 1 (negativos) estuvo compuesta por quienes no tenían un TUA, la Clase 2 por personas con un problema por consumo de alcohol, la Clase 3 por aquellos que padecían un TUA leve, la Clase 4 por niveles leves-moderados en Argentina, leves en España y moderados en Brasil, y la Clase 5 por graves en Argentina y Brasil, y moderados en España. Para denominar las clases se respetaron los nombres de cada nivel del TUA (negativo, un problema, leve, moderado, grave).

\section{Caracterización del Modelo de Cinco Clases Según Criterios de Diagnóstico, Niveles de Trastorno y CEEA}

En todos los contextos, tener un TUA leve, moderado o grave se relacionó con una probabilidad alta $(>\quad .5)$ de CEEA. Quienes padecían únicamente un problema por uso de alcohol en Argentina y España también tuvieron una alta probabilidad de CEEA; en cambio, en los estudiantes de Brasil fue moderada-baja. Para quienes no reportaron problemas, fue baja en
Argentina y Brasil, y moderada $(>$.3) en España (Tabla 3).

Las personas con un problema por consumo de alcohol en Argentina y España parecieron caracterizarse por una probabilidad moderada de negligencia en obligaciones prioritarias, mientras que en Brasil por una alta probabilidad de pérdida de control en el consumo. En Argentina, el nivel leve se caracterizó por una probabilidad moderada de tolerancia y consumo a pesar de problemas mentales/físicos mentales/físicos, y alta de pérdida de control y negligencia de actividades prioritarias. En estudiantes españoles, este nivel se caracterizó por una alta probabilidad de uso de alcohol en situaciones físicamente peligrosas y problemas interpersonales. En Brasil se observó una probabilidad moderada tolerancia, y alta de pérdida de control. En el nivel moderado, los estudiantes argentinos presentaban alta probabilidad de uso de alcohol en situaciones físicamente peligrosas y problemas interpersonales, y moderada de pérdida de control y negligencia de obligaciones prioritarias. Los estudiantes españoles, tuvieron una alta probabilidad de pérdida de control, y moderada de tolerancia, tiempo empleado en obtener/consumir alcohol, y negligencia de actividades prioritarias. Los universitarios brasileños evidenciaron una alta probabilidad de apetencia de la sustancia, pérdida de control, consumo a pesar de problemas mentales/físicos, y moderada de mayor consumo del pretendido, problemas interpersonales, y 
Conde, K. et al. / RACC, 2020, Vol. 12, №2, 1-10

negligencia de obligaciones prioritarias. Por último, el nivel grave se caracterizó por una probabilidad alta o moderada de endorsar todos los criterios, con excepción de apetencia de la sustancia y descuido de actividades en Argentina

y España, síntomas de abstinencia y mayor consumo del pretendido en España, y uso en situaciones físicamente peligrosas en Brasil (Tabla $3)$.

Tabla 3.

Probabilidades condicionales según criterios de diagnóstico, niveles de trastorno y consumo excesivo episódico, para un modelo de cinco clases, estudiantes universitarios de Argentina, España y Brasil

\begin{tabular}{|c|c|c|c|c|c|c|c|c|c|c|c|c|c|c|c|}
\hline \multirow[b]{2}{*}{ Variables } & \multicolumn{5}{|c|}{ Argentina $(N=2157)$} & \multicolumn{5}{|c|}{ España $(N=320)$} & \multicolumn{5}{|c|}{ Brasil $(N=149)$} \\
\hline & 1 & 2 & 3 & 4 & 5 & 1 & 2 & 3 & 4 & 5 & 1 & 2 & 3 & 4 & 5 \\
\hline CEEA & .26 & .50 & .72 & .73 & .91 & .32 & .56 & .65 & .78 & .92 & .12 & .28 & .38 & .80 & 1 \\
\hline \multicolumn{16}{|l|}{ Criterios diagnósticos } \\
\hline Síntomas de abstine & .00 & .07 & .20 & .05 & .44 & .00 & .07 & .06 & .18 & .08 & .00 & .00 & .21 & .27 & .33 \\
\hline Apete & .00 & .01 & .12 & .03 & .26 & .00 & .00 & .00 & .08 & .05 & .00 & .03 & .00 & .52 & .83 \\
\hline Tolerancia & .00 & .09 & .37 & .29 & .80 & .00 & .18 & .15 & .46 & .43 & .00 & .00 & .40 & .06 & 1 \\
\hline Descuido de & .00 & .00 & .03 & .00 & .24 & .00 & .00 & .00 & .02 & .06 & .00 & .00 & .13 & .07 & .83 \\
\hline Tien & .00 & .04 & .23 & .14 & .67 & .00 & .05 & .03 & .31 & .54 & .00 & .03 & .00 & .26 & .67 \\
\hline Mas & .00 & .01 & .07 & .05 & .50 & .00 & .04 & .00 & .09 & .09 & .00 & .00 & .00 & .32 & .67 \\
\hline Pér & .00 & .16 & .55 & .32 & .91 & .00 & .20 & .00 & .59 & .60 & .00 & .77 & 1 & .61 & .83 \\
\hline $\begin{array}{r}\text { Con } \\
\text { proble }\end{array}$ & .00 & .10 & .35 & .19 & .72 & .00 & .07 & .06 & .30 & .57 & .00 & .05 & .30 & .79 & 1 \\
\hline $\begin{array}{l}\text { Uso } \\
\text { físican }\end{array}$ & .00 & .00 & .00 & 1 & .80 & .00 & .00 & 1 & .00 & .86 & .00 & .03 & .13 & .20 & .17 \\
\hline Proble & .00 & .02 & .09 & 1 & .93 & .00 & .00 & 1 & .02 & 1 & .00 & .00 & .00 & .39 & .67 \\
\hline $\begin{array}{l}\text { Negligencia de obligaciones } \\
\text { prioritarias }\end{array}$ & .00 & .48 & .64 & .38 & .87 & .00 & .38 & .21 & .48 & .49 & .00 & .10 & .08 & .33 & .83 \\
\hline \multicolumn{16}{|l|}{ Trastorno por uso de alcohol } \\
\hline Negativo & 1 & .00 & .00 & .00 & .00 & 1 & .00 & .00 & .00 & .00 & 1 & .00 & .00 & .00 & .00 \\
\hline Un $p$ & .00 & 1 & 0 & 0 & .00 & .00 & 1 & .00 & .00 & .00 & .00 & 1 & .00 & .00 & .00 \\
\hline Leve & .00 & .00 & .83 & .53 & .00 & .00 & .00 & 1 & .85 & .00 & .00 & .00 & 1 & .00 & .00 \\
\hline Moder & .00 & .00 & .16 & .47 & .00 & .00 & .00 & .00 & .15 & .83 & .00 & .00 & .29 & .71 & .00 \\
\hline Grave & .00 & .00 & .00 & .00 & 1 & .00 & .00 & .00 & .00 & .17 & .00 & .00 & .00 & .00 & 1 \\
\hline
\end{tabular}

Nota. CEEA = consumo excesivo episódico de alcohol. Los ítems con probabilidad $>.3$ son destacados en negrita para su mejor identificación e interpretación

\section{Discusión}

En este estudio se examinaron las manifestaciones clínicas del TUA en tres grupos de estudiantes universitarios pertenecientes a tres contextos de consumo diferentes. Específicamente se estimó la probabilidad de que cada estudiante, agrupado según sus características de consumo, endorsara cada criterio de diagnóstico, añadiendo un ítem de consumo excesivo episódico de alcohol. Se obtuvieron cinco clases de bebedores en los tres contextos (países) para la población de estudiantes universitarios (argentinos, españoles y brasileños), cuyo consumo suele ser mayor al de la población general (Conde, Brandariz, \& Cremonte, 2016).

En general, el nivel grave de TUA se relacionó con una alta probabilidad de endorsar todos los criterios, en especial tolerancia, tiempo empleado en consumir/obtener la sustancia, pérdida de control, consumo continuado a pesar de los problemas, problemas interpersonales y negligencia de obligaciones prioritarias. La 
probabilidad de endorsar los criterios no fue similar en los criterios como síntomas de abstinencia, apetencia de la sustancia, descuido de actividades y uso en situaciones físicamente peligrosas. La posible explicación de estas observaciones se discute a continuación.

Si bien se ha encontrado que el TUA presenta una estructura unidimensional, también se ha encontrado que algunos síntomas son más severos que otros (Sher, Grekin, \& Williams, 2005). La presencia de síntomas de abstinencia es uno de los criterios considerados como más severos, indicador de una dependencia fisiológica e íntimamente relacionado a otros indicadores como el consumo matutino (Conde et al., 2016). Esto podría implicar que, de adoptarse una visión del TUA como una trayectoria en la cual hay momentos en los cuales la persona sufre síntomas más severos (Sher et al., 2005), los síntomas de abstinencia podrían ser propios de ellos. Ahora bien, es de notarse que los estudiantes españoles, en quienes el criterio síntoma de abstinencia tuvo una baja probabilidad, eran más jóvenes, y la categoría de TUA grave no estuvo representada como tal, pero sí se observaron dos clases de TUA leve. Futuras investigaciones sobre trayectorias de consumo podrían identificar si se debe a que una de estas categorías progresaría a niveles de mayor severidad. Lo mismo puede pensarse de los criterios descuido de actividades y apetencia de la sustancia, que sólo fueron altamente probables en los estudiantes de Brasil, donde el porcentaje de TUA grave fue mayor. No obstante, algunos estudios han alertado sobre el hecho de que el criterio apetencia de la sustancia no aportaría sustancialmente al diagnóstico de TUA (Casey, Adamson, Shevlin, \& McKinney, 2012).

Por otro lado, se observó que el espectro moderado-leve del TUA estaría caracterizado por criterios como tolerancia, pérdida de control, problemas interpersonales, y negligencia de obligaciones prioritarias. Es interesante notar que el criterio pérdida de control en el consumo fue característico de todos los niveles de TUA, siendo que desde un punto de vista teórico ha sido señalado como el síntoma principal del mismo (Conde et al., 2016). Las diferencias en la probabilidad de endorsar los criterios uso en situaciones físicamente peligrosas y consumo continuado a pesar de los problemas en los distintos niveles de severidad, se dieron sorprendentemente en los países donde existe un patrón húmedo o mediterráneo de consumo (i.e. Argentina y España). Este patrón se caracteriza por una alta aceptación del consumo de alcohol (Savic et al., 2016); de hecho, la prevalencia de consumo fue alta en ambos. Puede pensarse que, en estos contextos, la alta aceptación de la ingesta del consumo se traduce en el uso continuado y en situaciones de riesgo. En el criterio tiempo empleado en obtener/consumir alcohol, se observó una probabilidad moderada en estudiantes españoles. Respecto a lo anterior, en la literatura se ha reportado la práctica del botellón en los más jóvenes (conocida como previa en Argentina y pre-loading o pre-gaming en la literatura anglosajona), en donde se consume de alcohol de manera intensiva durante largos periodos de tiempo (Espejo, Cortés, del Río, Giménez, \& Gómez, 2012; Pilatti, Etkin, Parra, \& Pautassi, 2018). Estas prácticas en contextos donde el consumo es ampliamente aceptado, también podrían explicar lo observado respecto de la probabilidad de negligencia de obligaciones prioritarias en la clase que contenía a las personas con un problema por consumo de alcohol. Tanto en Argentina como en España, esta clase estuvo definida por este criterio y el CEEA. Junto con el consumo en general, y el consumo en exceso en la juventud, también podrían ser aceptables las consecuencias que repercuten en sus actividades de estudio, trabajo, etc. En cambio, en los estudiantes brasileños, con un patrón seco más similar al de los países anglosajones (Lima, Kerr-Correa, \& Rehm, 2013), la clase de personas con un problema no estuvo definida tanto por el CEEA, sino por la pérdida de control en el consumo. Una hipótesis que podría explicar este dato es que en contextos donde el consumo de alcohol no es aceptado, cualquier consumo podría ser visto como un consumo que no está siendo controlado por la persona, o también, mayor al esperado. En este sentido, es de notarse que el criterio mayor consumo del esperado fue más probable en el nivel moderado y grave de la muestra brasileña, algo que también fue encontrado en población general de Brasil y países con el mismo tipo de patrón cultural (Swift et al., 2016). Otros autores han encontrado que los jóvenes adultos reportan este criterio por razones sociales o culturales (Slade, Teesson, Mewton, Memedovic, \& Krueger, 2013).

Por último, el CEEA tuvo una alta probabilidad 
Conde, K. et al. / RACC, 2020, Vol. 12, №2, 1-10

en todos los niveles del TUA. Si bien se ha cuestionado su utilidad como criterio y la claridad de su relación con el TUA (McBride, Teesson, Baillie, \& Slade, 2011), este estudio aportaría datos a favor de la evidencia que sostiene que el patrón de consumo, o una medida de cantidad/frecuencia de consumo de alcohol, podría mejorar el diagnóstico en algunas poblaciones como la estudiada, o ser utilizada como marcador de TUA en reemplazo de medidas más complejas.

\section{Limitaciones}

Este estudio presenta una caracterización de los criterios y niveles de TUA, junto con un patrón de consumo de alcohol, en una población de riesgo; en adición, permite observar las manifestaciones del TUA en distintos contextos de consumo en muestras similares y con los mismos criterios. Adicionalmente, se presentan datos de países distintos a los de habla anglosajona, donde se suelen llevar a cabo este tipo de estudios, y con patrones de consumo culturales muy diferentes.

Sin embargo, en adición a las limitaciones propias de la selección y composición de las muestras, y a la imposibilidad de generalización de los resultados, se suman las propias de un diseño transversal y la pérdida de datos en algunas variables (que intentó ser abordada mediante la imputación múltiple). Lejos de restarle valor a los datos presentados, estas características junto con sus resultados remarcan la necesidad de realizar más investigaciones que aborden las hipótesis e interrogantes planteados (e.g. la interpretación cultural de los síntomas y el desarrollo del TUA). Además, da indicios de la posible aplicación de una medida de cantidad/frecuencia de consumo de alcohol para el tamizaje y rápida identificación de problemas por uso de alcohol en poblaciones similares a la estudiada.

\section{Agradecimientos}

Este trabajo ha sido realizado con el aporte del Consejo Nacional de Investigaciones Científicas y Técnicas y a la Universidad Nacional de Mar del Plata por haber contribuido parcialmente a la financiación de este proyecto a través de una Beca de Finalización de Doctorado y una Beca de Iniciación a la Investigación, respectivamente, otorgadas a la Dra. Karina
Conde.

\section{Conflictos de interés}

Declaramos no poseer intereses secundarios que pudieran incidir en este trabajo.

\section{Referencias}

American Psychiatric Association. (2000). Diagnostic and statistical manual-text revision (DSM-IV-TRim, 2000). Washington: American Psychiatric Association.

American Psychiatric Association. (2013). The diagnostic and statistical manual of mental disorders: DSM 5. Philadelphia: American Psychiatric Association.

Bond, J., Ye, Y., Cherpitel, C. J., Borges, G., Cremonte, M., Moskalewicz, J., \& Swiatkiewicz, G. (2012). Scaling properties of the combined ICD-10 dependence and harms criteria and comparisons with DSM-5 alcohol use disorder criteria among patients in the emergency department. Journal of Studies on Alcohol and Drugs, 73(2), 328-336. doi: 10.15288/jsad.2012.73.328

Borges, G., Ye, Y., Bond, J., Cherpitel, C. J., Cremonte, M., Moskalewicz, J., ... Rubio-Stipec, M. (2010). The dimensionality of alcohol use disorders and alcohol consumption in a cross-national perspective. Addiction, 105(2), 240-254. doi: 10.1111/j.1360-0443.2009.02778.x

Buuren, S., \& Groothuis-Oudshoorn, K. (2011). Mice: Multivariate imputation by chained equations in R. Journal of Statistical Software, 45(3), 1-68. doi: 10.18637/jss.v045.i03

Caldeira, K. M., Kasperski, S. J., Sharma, E., Vincent, K. B., O'Grady, K. E., Wish, E. D., \& Arria, A. M. (2009). College students rarely seek help despite serious substance use problems. Journal of Substance Abuse Treatment, 37(4), 368-378. doi: 10.1016/j.jsat.2009.04.005

Casey, M., Adamson, G., Shevlin, M., \& McKinney, A. (2012). The role of craving in AUDs: Dimensionality and differential functioning in the DSM-5. Drug and Alcohol Dependence, 125(2), 75-80. doi: 10.1016/j.drugalcdep.2012.03.019

Conde, K., Brandariz, R. A., \& Cremonte, M. (2016). Problemas por uso de alcohol en estudiantes secundarios y universitarios. Revista Chilena de Neuro-Psiquiatría, 54(2), 84-93. doi: 10.4067/S0717-92272016000200002

Conde, K., \& Cremonte, M. (2015). Calidad de los datos de encuestas sobre consumo de alcohol en estudiantes universitarios. Cadernos de Saúde Pública, 31(1), 39-47. doi: 10.1590/0102311X00061114

Dawson, D. A. (2000). Drinking patterns among individuals with and without DSM-IV alcohol use 
disorders. Journal of Studies on Alcohol, 61(1), 111-120. doi: 10.15288/jsa.2000.61.111

Espejo, B., Cortés, M. T., del Río, B. M., Giménez, J. A., \& Gómez, C. (2012). Traits that define the different alcohol intensive consume type during the practice of "Botellon". The Spanish Journal of Psychology, 15(1), 256-264. doi: 10.5209/rev SJOP.2012.v15.n1.37318

Gilbert, P. A., \& Marzell, M. (2018). Characterizing a hidden group of at-risk drinkers: Epidemiological profiles of alcohol-use disorder diagnostic orphans. Substance Use \& Misuse, 53(8), 1239-1251. doi: 10.1080/10826084.2017.1402057

Grabitz, M., Behrendt, S., Klotsche, J., Buehringer, G., Lieb, R., \& Wittchen, H. U. (2012). Ten-year stability and variability, drinking patterns, and impairment in community youth with diagnostic orphan status of alcohol dependence. Addictive Behaviors, 37(4), 399-406. doi: 10.1016/j.addbeh.2011.11.031

Hagman, B. T. (2018). Characteristics of DSM-5 alcohol use disorder diagnostic orphans in college: An overlooked group of drinkers. Addictive Disorders \& their Treatment, 17(1), 1-12. doi: 10.1097/ADT.0000000000000116

Hingson, R. W., Zha, W., \& Weitzman, E. R. (2009). Magnitude of and trends in alcohol-related mortality and morbidity among US college students ages 1824, 1998-2005. Journal of Studies on Alcohol and Drugs, Supplement, 16(1), 12-20. doi: 10.15288/jsads.2009.s16.12

Jackson, K., Bucholz, K., Wood, P., Steinley, D., Grant, J., \& Sher, K. (2014). Towards the characterization and validation of alcohol use disorder subtypes: Integrating consumption and symptom data. Psychological Medicine, 44(1), 143-159. doi: 10.1017/S0033291713000573

Karam, E., Kypri, K., \& Salamoun, M. (2007). Alcohol use among college students: An international perspective. Current Opinion in Psychiatry, 20(3), 213-221. doi: 10.1097/YCO.0b013e3280fa836c

Kessler, R. C., \& Üstün, T. B. (2004). The world mental health $(\mathrm{WMH})$ survey initiative version of the world health organization (WHO) composite international diagnostic interview (CIDI). International Journal of Methods in Psychiatric Research, 13(2), 93-121. doi: $10.1002 / \mathrm{mpr} .168$

Li, T., Hewitt, B. G., \& Grant, B. F. (2007). Is there a future for quantifying drinking in the diagnosis, treatment, and prevention of alcohol use disorders? Alcohol and Alcoholism, 42(2), 57-63. doi: 10.1093/alcalc/agl125

Lima, M. C., Kerr-Correa, F., \& Rehm, J. (2013). Alcohol consumption pattern and coronary heart disease risk in metropolitan Sao Paulo: Analyses of GENACIS project. Brazilian Journal of Epidemiology, 16(1), 49-57. doi: 10.1590/\$1415-

\section{$790 \times 2013000100005$}

Linzer, D. A., \& Lewis, J. B. (2011). poLCA: An R package for polytomous variable latent class analysis. Journal of Statistical Software, 42(10), 129. doi: $10.18637 /$ jss.v042.i10

McBride, O., Teesson, M., Baillie, A., \& Slade, T. (2011). Assessing the dimensionality of lifetime DSM-IV alcohol use disorders and a quantityfrequency alcohol use criterion in the Australian population: A factor mixture modelling approach. Alcohol and Alcoholism, 46(3), 333-341. doi: 10.1093/alcalc/agr008

Pilatti, A., Etkin, P., Parra, E. U., \& Pautassi, R. (2018). De fiesta antes de la fiesta: relación entre esta práctica de consumo de alcohol con los problemas derivados del uso de alcohol en jóvenes argentinos. Health \& Addictions, 18(1), 5-16. doi: 10.21134/haaj.v18i1.318

Saha, T. D., Stinson, F. S., \& Grant, B. F. (2007). The role of alcohol consumption in future classifications of alcohol use disorders. Drug and Alcohol Dependence, 89(1), 82-92. doi: 10.1016/j.drugalcdep.2006.12.003

Savic, M., Room, R., Mugavin, J., Pennay, A., \& Livingston, M. (2016). Defining "drinking culture": A critical review of its meaning and connotation in social research on alcohol problems. Drugs: Education, Prevention and Policy, 23(4), 270-282. doi: 10.3109/09687637.2016.1153602

Sher, K. J., Grekin, E. R., \& Williams, N. A. (2005). The development of alcohol use disorders. Annual Review of Clinical Psychology, 1(1), 493-523. doi: 10.3109/09687637.2016.1153602

Slade, T., Teesson, M., Mewton, L., Memedovic, S., \& Krueger, R. F. (2013). Do young adults interpret the DSM diagnostic criteria for alcohol use disorders as intended? A cognitive interviewing study. Alcoholism: Clinical and Experimental Research, 37(6), 1001-1007. doi: 10.1111/acer.12063

Slutske, W. S. (2005). Alcohol use disorders among US college students and their non-college-attending peers. Archives of General Psychiatry, 62(3), 321327. doi: 10.1001/archpsyc.62.3.321

Swift, W., Slade, T., Carragher, N., Coffey, C., Degenhardt, L., Hall, W., \& Patton, G. (2016). Adolescent predictors of a typology of DSM-5 alcohol use disorder symptoms in young adults derived by latent class analysis using data from an Australian cohort study. Journal of Studies on Alcohol and Drugs, 77(5), 757-765. doi: 10.15288/jsad.2016.77.757

Zeigler, D. W., Wang, C. C., Yoast, R. A., Dickinson, B. D., McCaffree, M. A., Robinowitz, C. B., \& Sterling, M. L. (2005). The neurocognitive effects of alcohol on adolescents and college students. Preventive Medicine, 40(1), 23-32. doi: 
Conde, K. et al. / RACC, 2020, Vol. 12, №2, 1-10

10.1016/j.ypmed.2004.04.044

Zhang, Z., Abarda, A., Contractor, A. A., Wang, J., \& Dayton, C. M. (2018). Exploring heterogeneity in clinical trials with latent class analysis. Annals of Translational Medicine, 6(7), 119 . doi: 10.21037/atm.2018.01.24 